\title{
Scarring at Donor Sites after Split-Thickness Skin Graft: A Prospective, Longitudinal, Randomized Trial
}

Matilda Karlsson, Moustafa Elmasry, Ingrid Steinvall, Folke Sjöberg, Pia Olofsson and Johan Thorfinn

The self-archived postprint version of this journal article is available at Linköping University Institutional Repository (DiVA):

http://urn.kb.se/resolve?urn=urn:nbn:se:liu:diva-171957

N.B.: When citing this work, cite the original publication.

Karlsson, M., Elmasry, M., Steinvall, I., Sjöberg, F., Olofsson, P., Thorfinn, J., (2020), Scarring at Donor Sites after Split-Thickness Skin Graft: A Prospective, Longitudinal, Randomized Trial,

Advances in Skin \& Wound Care, 33(12), . https://doi.org/10.1097/01.ASW.0000720256.45983.28

Original publication available at:

https://doi.org/10.1097/01.ASW.0000720256.45983.28

Copyright: Lippincott, Williams \& Wilkins

http://www.lww.com/ 


\begin{abstract}
Objective: The aim of this study was to investigate if previous findings on the association between dressing treatments and subjective opinion on final donor site scar outcome using the patient scale of POSAS could be confirmed objectively. The previous study showed that patients dressed with hydrofiber covered with film were more satisfied with their donor site scars than patients dressed with porcine xenograft or polyurethane foam.
\end{abstract}

Methods: Scar outcome measurements were assessed by a blinded observer using POSAS and the device: Cutometer ${ }^{\circledR}$ dual MPA 580 to measure the viscoelasticity of the skin.

Results: A total of 17 participants were included in this study, five came from the hydrofiber group, six from the polyurethane foam, and six from porcine xenograft group. There were no significant differences between dressing groups in any of the items on POSAS or in the viscoelasticity measurements done with the Cutometer.

Conclusion: The investigators could not confirm previous findings of association between dressing treatment and the long-term scarring of the donor site. No associations between donor sites' healing times and final scarring were found. Hypopigmentation was reported in 15/17 donors sites evaluated.

Keywords: STSG, donor site, dressing, scar, Cutometer, POSAS 


\section{Introduction}

This is the third and last part of a clinical project concerning the treatment of split thickness skin graft donor sites. The first part of the project was a randomized clinical trial (RCT) were study participants were randomly assigned to treatment with either hydrofiber covered with film, non-adhesive polyurethane foam, or porcine xenograft on their donor site. ${ }^{1}$ Results from this study indicated that donor sites treated with hydrofiber and porcine xenograft healed significantly faster than those treated with polyurethane foam. As the hydrofiber was the most comfortable, easy to use, and costeffective, it was implemented as the standard of care for donor sites at the current department.

In the second part of the project the long-term scar outcomes of the donor sites included in the RCT were investigated. ${ }^{2}$ As a longer healing time has been shown to be a predictor for more severe donor site scarring ${ }^{3,4}$ it was hypothesized that patients treated with polyurethane foam, would be the most unsatisfied with their scars, if they had any donor site scars. Study participants were asked to evaluate their scars using the "Patients part" of the Patient and Observer Scar Assessment Scale (POSAS) eight years after inclusion in the RCT. The Patient Scale contains six questions that address the items: pain, itching, color, stiffness, thickness and relief. Each of the six items has a 10-point range (1 - 10), with 10 indicating the worst imaginable scar or sensation. The lowest score, "1" corresponds to the normal skin. The patient can also score their "Overall Opinion"” on the scar compared to normal skin with a 10 -step scale, with 10 indicating a scar very different from normal skin. ${ }^{5}$

After analyzing the data collected in the second part, the investigators could conclude that the fast and moist healing seen with hydrofiber seemed to result in significantly more satisfied patients - and members of the polyurethane foam group, as hypothesized, were significantly more unsatisfied with their donor site scars. Surprisingly the dressing associated with the fastest wound healing (the porcine xenograft) also had the poorest long-term outcome for scarring, according to the patients' opinion ${ }^{2}$. This contradicts what is claimed to be the relation between duration of healing and donor site scarring. ${ }^{3,4}$

In this last, third part, the aim was to investigate if the previous findings could be confirmed by an evaluation done by a blinded observer using the Observer part of the POSAS ${ }^{4,5}$ and the device; Cutometer dual MPA 580 (Courage and Khazaka Electronic $\mathrm{GmbH}$, Cologne, Germany), measuring viscoelasticity of the donor site scar. ${ }^{6}$ 


\section{Patients and methods}

\section{Patients}

All patients originally included in the RCT study "Dressing the Split-Thickness Skin Graft Donor Site: A Randomized Clinical Trial" and who later participated in the subjective scar follow up "Split thickness skin graft donor sites: Eight years follow-up of patient-reported scar outcome - A prospective longitudinal randomized trial" ${ }^{2}$ were contacted by mail and asked to participate in this last part of the project. In this part, patients were informed about the study and asked to visit the current department for an assessment of their donor site scars. Patients were asked to "tick the box" Yes, I want to be scheduled for a donor sites scar assessment or No, I do not wish to participate in the study, sign and date and then send the answer back in a preaddressed envelope. Patients who were willing to participate were scheduled for a visit to an occupational/scar therapist responsible for scar follow ups.

\section{Assessment of the scar}

\section{The Patient and Observer Scar Assessment Scale (POSAS)}

An occupational therapist (using POSAS in clinical routine) assessed the donor site scar using the "Observer part" of the POSAS (Supplemental Fig. 1). ${ }^{5}$ The items included are: vascularity, pigmentation, thickness, relief, pliability, and surface area. For each item on the scale a score of 1 indicates "best possible" and 10 "worst possible" outcome. To be able to specify abnormalities each item can also be "categorized". The best possible total score is 6 and the worst possible score 60 . There is also an item called "overall opinion", which was not included in this study as it is not a part of the clinical routine at the current department.

\section{The Cutometer}

The Cutometer dual MPA 580 was used for assessment of the viscoelastic properties of both the donor site and (contralateral) uninjured skin for comparison (called controls). The Cutometer has been shown to reproducibly calculate the viscoelastic properties by measuring the deformation of the skin in millimeters under a controlled vacuum. ${ }^{6,7}$

In this study, a probe with a diameter of $6 \mathrm{~mm}$ was used (as seen in similar studies). ${ }^{6,8-}$ 10 The device exerted a controlled vacuum of 450 mbar on the surface of the skin for a period of two seconds ("On-time"), followed by two seconds of normal pressure ("Offtime"). Each measurement was taken in the center of the area of interest and was automatically repeated three times. The resistance of the skin to being sucked up by negative pressure (firmness) and its ability to return into its original position (elasticity) 
were displayed as curves. The following values were given R0-R9, F0 and F1, and Q0Q3. In this study, the following variables R0, R2 and F1 will be described and results displayed, as seen in a similar, published article. ${ }^{11}$

\section{Description of Cutometer variables}

$\boldsymbol{R O}(\mathrm{Uf})=$ First maximum amplitude, the highest point of the first curve. This has an implication for the firmness of the skin. This variable represents the passive behavior of the skin under force. The lower the value, the higher the firmness of the skin. The result for the curve is given as distance in millimeters.

$\boldsymbol{R} 2(\mathrm{Ua} / \mathrm{Uf})=$ The portion between the maximum amplitude and the ability to return to the original position. The closer the value is to $1(100 \%)$ the more elastic the curve. Results for the curve are given as percentages (\%).

$\boldsymbol{F 1}(R 0 \mathrm{x}$ relaxation time $)=$ Area within the rectangle. This area is deducted from the total area (rectangle $=$ maximum amplitude and time). A completely elastic material will show the complete area (total area and F1 are the same), the closer the value for total area minus F1 to 0 , the more elastic the surface studied.

\section{Evaluation of Hypertrophic Scarring}

Regarding hypertrophic scarring (HTS) to models previous suggested in scar studies were used. ${ }^{12-14}$

1) Scars over the median score for the entire group are likely to be hypertrophic.

2) Scars that score higher than $1(1=$ not high at all $)$ on the item "thickness" are likely to be hypertrophic if categorized as "thicker".

The blinded Observer was asked to state if the scar was hypertrophic in their opinion.

\section{Statistical analysis}

Statistical analysis was done using STATA (STATA version 12.0, Stata Corp. LP College Station, TX, USA). Descriptive data are given as median (range) unless otherwise stated. To detect any significant differences in the scores of the POSAS items between the three dressings the Chi squared test was used (Fisher's exact test was used when appropriate). As all items showed ties (the same score-values given for several donor sites) the Kruskal Wallis one-way ANOVA could not be used. To detect any 
significant differences between two groups the Mann-Whitney $U$ test was used (and $p$ values were corrected for multiple comparison). To detect differences between the three groups for the Cutometer values (R0-9 and Q1-3) the one-way ANOVA with Bonferroni correction was used. For comparison within groups for values on the Cutometer the Student's t-test was used (and Mann Whitney U test when appropriate). Probabilities of less than 0.05 were accepted as significant.

The initial part of the study was reviewed and approved by the Regional Ethics Review Board in 2008 (M130-08), the first follow up study in 2017 (Dnr. 2017/43-41), and this last part was approved in 2018 (2018/212-31).

\section{Results}

\section{Description of the study group}

Of the 27 participants in the second part of the study, two had died, six (with a total of 8 donor sites) chose not to participate and two did not answer the letter. The remaining 17 were included in the Observer follow up, approximately 10 years after the initial RCT. Of the 17 participants, five came from the hydrofiber group, six from the polyurethane foam, and six from porcine xenograft group. The groups were similar in age, sex distribution and, unlike the previous studies, also in healing time (Table 1.)

\section{Observer opinion on scarring with POSAS}

There were no significant differences between the three dressing groups for the total POSAS score $(\mathrm{p}=0.968)$ or in any of the POSAS items according to the Observer (Supplemental Table 1). The Observer scored over all low for all items. The median score was 1 (= like normal skin) for the items vascularity, thickness, relief, and pliability. Only pigmentation and surface area had a median higher than 1. Regarding pigmentation, 16 of the 17 donor sites showed abnormal pigmentation, 15 of these were categorized as hypo-pigmented, and one as hyper-pigmented. As for surface area, scores on this item indicated that the abnormal pigmentation was seen at most of the original donor site area. All POSAS scores given by the Observer and the total POSAS score given by the Patient in the previous study are shown in Supplemental Table 2. 


\section{Healing time and degree of scarring}

When regrouping participants, regardless of their previous dressing treatment, according to their healing time (categorized as follows: $0=14$ days or shorter, $1=15$ to 21 days, and $2=$ longer than 21 days) no differences were seen for any of the POSAS items.

\section{The Cutometer}

No significant differences were detected for any of the Cutometer values assessed (R0R9, F0, F1, and Q0-Q3). When comparing uninjured skin (control) with donor sites within the groups, no differences were detected other than in the porcine xenograft group for the value F1 (elasticity) where the donor site showed a lower mean ( $F 1=0.13,95 \%$ C.I 0.11 to 0.15$)$ indicating more elastic skin than the control $(\mathrm{F} 1=0.15,95 \%$ C.I 0.14 to 0.17$),(\mathrm{p}=0.022)$.

Regarding controls (uninjured skin), there were no differences between groups for any of the values assessed with the Cutometer, nor were there any difference when comparing donor site values between the groups (Table 2). Also, when comparing mean differences (donor site values - control values) between groups, none was found (Supplemental Table 3).

\section{Healing time and Cutometer values}

When ranking donor sites after healing time (described in the Method section) no significant differences in any of the Cutometer values could be seen (Supplemental Table 4).

A box-plot (showing median, IQR and range) for R0 and R2 values for uninjured skin (controls) and the different treatment groups can be seen in Figs.1 and 2. In Figs. 3 and 4, donor site values for R0 and R2 are presented with respect to healing time instead of dressing treatment. As R2 and F1 are both measurements of elasticity of the skin F1 values are displayed in Supplemental figures 2 and 3.

Range or "whiskers" in the box-plots extend to 1.5 times the height of the box or, if no case/row has a value in that range, to the minimum or maximum values. As values of R0, R2 and F1 seems normally distributed (within bell shaped normal curve) the whiskers cover approximately $95 \%$ of the values observed (if not minimum to maximum is displayed than $100 \%$ is covered). In the figures extreme values/outliers are shown with an asterisk (extreme values more than three times the height of the box). 


\section{Hypertrophic scarring}

Using Model 1. seven patients had a total score higher than 16 (median for the entire group), two from the hydrofiber group, two from the polyurethane foam, and three from the porcine xenograft. The main reason for higher total scores was a high deviation from normal skin regarding pigmentation, as described earlier, (Supplemental Table 1 and 2).

Using Model 2. Three patients had a thickness score higher than 1 but were all categorised as "thinner" than normal, not indicating HTS but the opposite.

According to the blinded Observer, no patients presented with HTS, thereby confirming that results from the second model.

\section{Discussion}

In this study, a long-term follow up/evaluation for donor sites scars treated with three different dressings was done by a blinded observer using the POSAS observer tool and the Cutometer to compare donor site elasticity and firmness between the different groups and controls. Previous findings on associations between dressing treatment and scar outcome could not be confirmed, ${ }^{2}$ nor could a relationship between longer healing times and scar outcome be confirmed. ${ }^{3,4}$

\section{POSAS observer interpretation}

One possible explanation for not being able to confirm previous results could be that the Observer did not score "Itch" and "Pain" as these items might have affected the results in the previous study. As displayed in the previous study all study participants scored 1 on these two items, indicating that their effect on the results would have been minimal. ${ }^{2}$ Another more likely explanation is that the previous significant difference in healing time between groups was not seen in the group that had the POSAS Observer's evaluation. The patients with longer healing times did not return, for unknown reasons.

The total scores given by participants in the second part and who did not return for assessment by the Observer was examined. Of the six patients in the hydrofiber group that did not return all but one had lower "subjective" total scores than the returners ( 7 , $7,7,8,9$, and 10) meaning that the more unsatisfied patients from this group returned. For the two other dressing groups, the opposite was seen, the "non-returners" had given high subjective total scores meaning that the more satisfied patients returned for the observer assessment from these groups. For the polyurethane foam group, the "nonreturners" scored 12, 20, and 28, and the porcine xenograft group scored 9, 23, and 41. As the number of observations were small, this might have had an impact on the results. 
The POSAS item pigmentation had an important role in the scoring done by the patients; it was also the only item that affected the total score for the Observer. As donor sites were categorized as hypo-pigmented in 15 out of a total of 17 donor sites, it can be concluded that this seems to be a "common case" for the group. As skin grafts are harvested at a thickness of about $0.2-0.3 \mathrm{~mm}$, epidermal skin appendages containing melanocytes should be present in the donor sites to enable pigmentation of the scar. A previous (small) study on donor sites that measured the amount of melanin at the scar (using a Mexameter) concluded that even though melanin levels were lower at donor sites than in controls, they were not significantly so. Visual hypo-pigmentation was reported in this study, but with a lower incidence than reported here. ${ }^{11}$

In 2001, Velangi et al. presented a study in which biopsies were taken from long-term hypo-pigmented scars in human and it was found that there were no differences in melanocyte number or activity in pale scars than healthy skin in the same individual. Instead, their theory was that the pale outcome was related to decreased vascularity, or a second possibility was that the abnormal organization of collagen within the dermis, or epidermal change resulted in a greater reflection of light back towards the observer. ${ }^{15}$

\section{Skin elasticity evaluated by Cutometer}

Regarding the values given by the Cutometer, interpretations have to be made cautiously, because the number of observations are small and the use of the Cutometer has only recently been implemented at the department. It is still interesting, though, that the firmness and elasticity of the hydrofiber group seems to be the closest to the controls of uninjured skin. Even more interesting, is that the donor site scars seem to be more elastic than their controls. The polyurethane foam scars (with the longest healing times) seemed to be the most elastic. As for differences between un-injured skin and donor sites, only the porcine xenograft group showed significant differences for one elasticity measurement (F1) in this study, in which donor sites were more elastic than controls. Data from a similar study showed significant differences between uninjured skin and donor sites in firmness (R0) in which donor sites were less firm than controls. ${ }^{8}$ In this study, the polyurethane foam and porcine xenograft groups were less firm than controls, but not significantly so.

Looking at firmness (R0), similar values could be seen for controls, hydrofiber, and healing times under 14 days. Longer healing time (more than 21 days) seemed to provide more elastic scars and less firmness. No significant association between viscoelastic measurements and healing time, could be find, confirming the results from a similar study that was published in $2006 .{ }^{8}$ 
As this is a new method of evaluation, the investigators lack a good explanation for these findings, but further studies are recommend using this tool on a greater number of subjects.

None of the donor sites presented with HTS, according to the Observer. A recently published article investigating the long-term prevalence of HTS in donor sites in children (after operations for burns) reports persistent HTS in $34 \%$ (mean follow up was 2.4 years).These findings might be related to potential differences in childhood and adult scarring, because young age has been suggested as a risk factor for HTS. ${ }^{16}$

\section{Limitations of the study}

First, despite being a prospective, randomized trial, this study had a small number of patients, many of whom were lost to follow up. Further scar follow-up studies after RCT studies of dressing treatments are recommended. All scar follow ups were performed by a single observer. Adding repeated measurements or multiple observers could have affected the results.

There is no consensus on which POSAS score indicates hypertrophic scarring. This lack of consensus about definitions of pathological scarring in general, and hypertrophic scarring in particular, is a drawback of all the commonly-used scar scales. Using the suggested model where scars over the median score for the entire group are likely to be hypertrophic rendered 7 hypertrophic patients, whilst the other model and the observer opinion gave no hypertrophic scars. Looking closer at the scar scores for the item thickness only three patients had scores above 1 and they were all categorized as "thinner" indicating that the reason for a higher score than the median was not hypertrophic scarring. This highlights the drawback with using this model on POSAS scores. The models were originally used for the Vancouver scar scale (VSS) and results must therefore be interpreted with caution.

Approaches have been made, for example, to modify the POSAS scale to distinguish pathological scarring from non-pathological scarring. ${ }^{17}$ By doing so, prevalence could be assessed, and the efficacy of treatments could be better evaluated.

\section{Conclusion}

The main finding of this study was that no association between the dressing treatment and the long-term scarring of the donor site, using POSAS or a Cutometer, could be found. As in the previous study, no association between longer healing times and higher scar scores were found. Fifteen out of the 17 donor sites showed hypo-pigmentation approximately 10 years after the harvest of skin grafts, which warrants future studies on the subject. 


\section{References}

1. Karlsson M, Lindgren M, Jarnhed-Andersson I, et al. Dressing the split-thickness skin graft donor site: a randomized clinical trial. Adv Skin Wound Care, 2014. 27(1): p. 20-5.

2. Karlsson M, Elmasry M, Steinvall I, et al. Scarring At Donor Sites After Split-Thickness Skin Graft: A Prospective, Longitudinal, Randomized Trial. Adv Skin Wound Care, 2018. 31(4): p. 183-188.

3. Rotatori RM, Starr B, Peake M, et al. Prevalence and Risk Factors for Hypertrophic Scarring of Split Thickness Autograft Donor Sites in a Pediatric Burn Population. Burns, 2019. 45(5): p. 1066-1074.

4. Werdin F, Tenenhaus M, Becker M, et al. Healing Time Correlates With the Quality of Scaring: Results From a Prospective Randomized Control Donor Site Trial. Dermatol Surg, 2018. 44(4): p. 521-527.

5. Draaijers LJ, Tempelman FRH, Botman YAM, et al. The Patient and Observer Scar Assessment Scale: A Reliable and Feasible Tool for Scar Evaluation. Plastic and Reconstructive Surgery, 2004. 113(7): p. 1960-1965.

6. Draaijers LJ, Botman YA, Tempelman FR, et al. Skin elasticity meter or subjective evaluation in scars: a reliability assessment. Burns, 2004. 30(2): p. 109-14.

7. Everett JS and Sommers MS. Skin viscoelasticity: physiologic mechanisms, measurement issues, and application to nursing science. Biol Res Nurs, 2013. 15(3): p. 338-46.

8. Rennekampff HO, Rabbels J, Reinhard V, et al. Comparing the Vancouver Scar Scale with the cutometer in the assessment of donor site wounds treated with various dressings in a randomized trial. J Burn Care Res, 2006. 27(3): p. 345-51.

9. Lee KC, Bamford A, Gardiner F, et al. Investigating the intra- and inter-rater reliability of a panel of subjective and objective burn scar measurement tools. Burns, 2019. 45(6): p. 13111324.

10. Busche MN, Thraen AJ, Gohritz A, et al. Burn Scar Evaluation Using the Cutometer(R) MPA 580 in Comparison to "Patient and Observer Scar Assessment Scale" and "Vancouver Scar Scale". J Burn Care Res, 2018. 39(4): p. 516-526.

11. Schulz A, Rothermund I, Lefering R, et al. Long-term Scar Quality after Treatment of Standardized Partial-Thickness Skin Graft Donor Sites. Adv Skin Wound Care, 2018. 31(3): p. 109-117.

12. Thompson CM, Hocking AM, Honari S, et al. Genetic risk factors for hypertrophic scar development. J Burn Care Res, 2013. 34(5): p. 477-82.

13. Thompson CM, Sood RF, Honari S, et al. What score on the Vancouver Scar Scale constitutes a hypertrophic scar? Results from a survey of North American burn-care providers. Burns, 2015. 41(7): p. 1442-8.

14. Goei $\mathrm{H}$, van der Vlies $\mathrm{CH}$, Tuinebreijer WE, et al. Predictive validity of short term scar quality on final burn scar outcome using the Patient and Observer Scar Assessment Scale in patients with minor to moderate burn severity. Burns, 2017. 43(4): p. 715-723. 
15. Velangi SS and Rees JL. Why are scars pale? An immunohistochemical study indicating preservation of melanocyte number and function in surgical scars. Acta Derm Venereol, 2001. 81(5): p. 326-8.

16. Gangemi EN, Gregori D, Berchialla P, et al. Epidemiology and risk factors for pathologic scarring after burn wounds. Arch Facial Plast Surg, 2008. 10(2): p. 93-102.

17. Fearmonti RM, Bond JE, Erdmann D, et al. The modified Patient and Observer Scar Assessment Scale: a novel approach to defining pathologic and nonpathologic scarring. Plast Reconstr Surg, 2011. 127(1): p. 242-7. 


\section{Tables and figures}

\begin{tabular}{lcccc}
\hline \multicolumn{5}{c}{ Table 1. Baseline characteristics of included patients by treatment group } \\
\hline \\
$\begin{array}{c}\text { Hydrofiber } \\
(\mathrm{n}=5)\end{array}$ & $\begin{array}{c}\text { Polyurethane foam } \\
(\mathrm{n}=6)\end{array}$ & $\begin{array}{c}\text { Porcine xenograft } \\
(\mathrm{n}=6)\end{array}$ & $\mathrm{p}$ values \\
Age (years) & $70(45-82)$ & $71(45-81)$ & $64(30-80)$ & 0.954 \\
Sex (M/F) & $3 / 2$ & $4 / 2$ & $3 / 3$ & 1.0 \\
Healing time & & & & \\
within 14 days & 4 & 2 & 3 & \\
within 21 days & 0 & 2 & 2 & \\
$>21$ days & 1 & 2 & 1 & 0.683 \\
\hline
\end{tabular}

Data are given in median and range (in brackets) or number when appropriate.

Table 2. Cutometer measurements on donor sites grouped by dressing treatment

\begin{tabular}{lcccc}
\hline & Hydrofiber & Polyurethane foam & Porcine xenograft & p values \\
R0 & $1.10(0.23)$ & $1.10(0.13)$ & $1.07(0.14)$ & 0.942 \\
R2 & $0.82(0.09)$ & $0.77(0.09)$ & $0.79(0.08)$ & 0.619 \\
F1 & $0.15(0.03)$ & $0.11(0.03)$ & $0.13(0.02)$ & 0.155 \\
\hline
\end{tabular}

Data are mean values and standard deviations. Lower R0 indicates higher firmness, higher R2 indicates higher elasticity, and lower F1 indicates higher elasticity. 


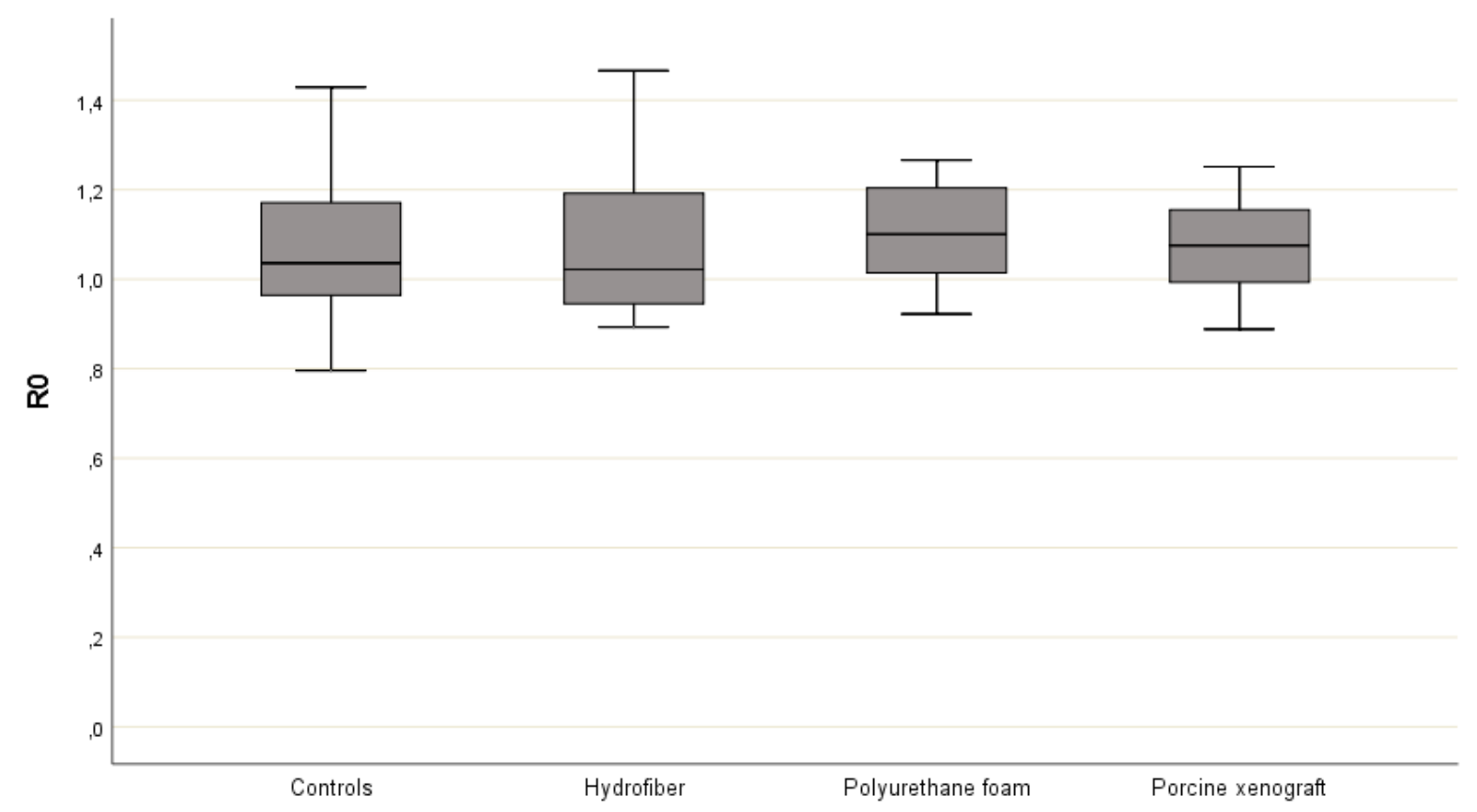

Figure1. R0 values for all controls and the three dressing groups. The lower the value, the higher the firmness of the skin. Median, IQR and range are displayed.

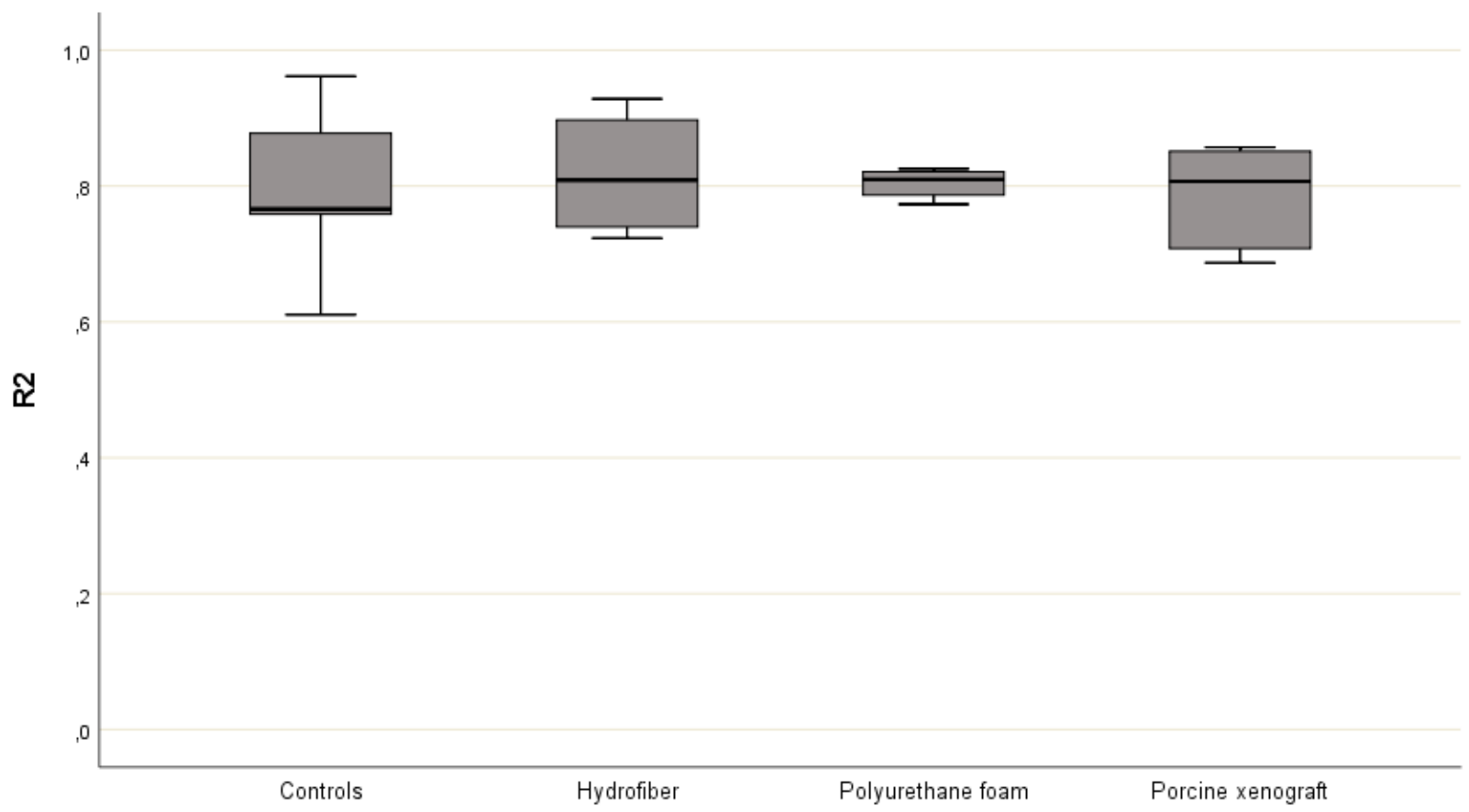

Figure 2. R2 values for all controls and the three dressing groups. The closer the value is to 1 (100\%), the more elastic the skin. Median, IQR and range are displayed 


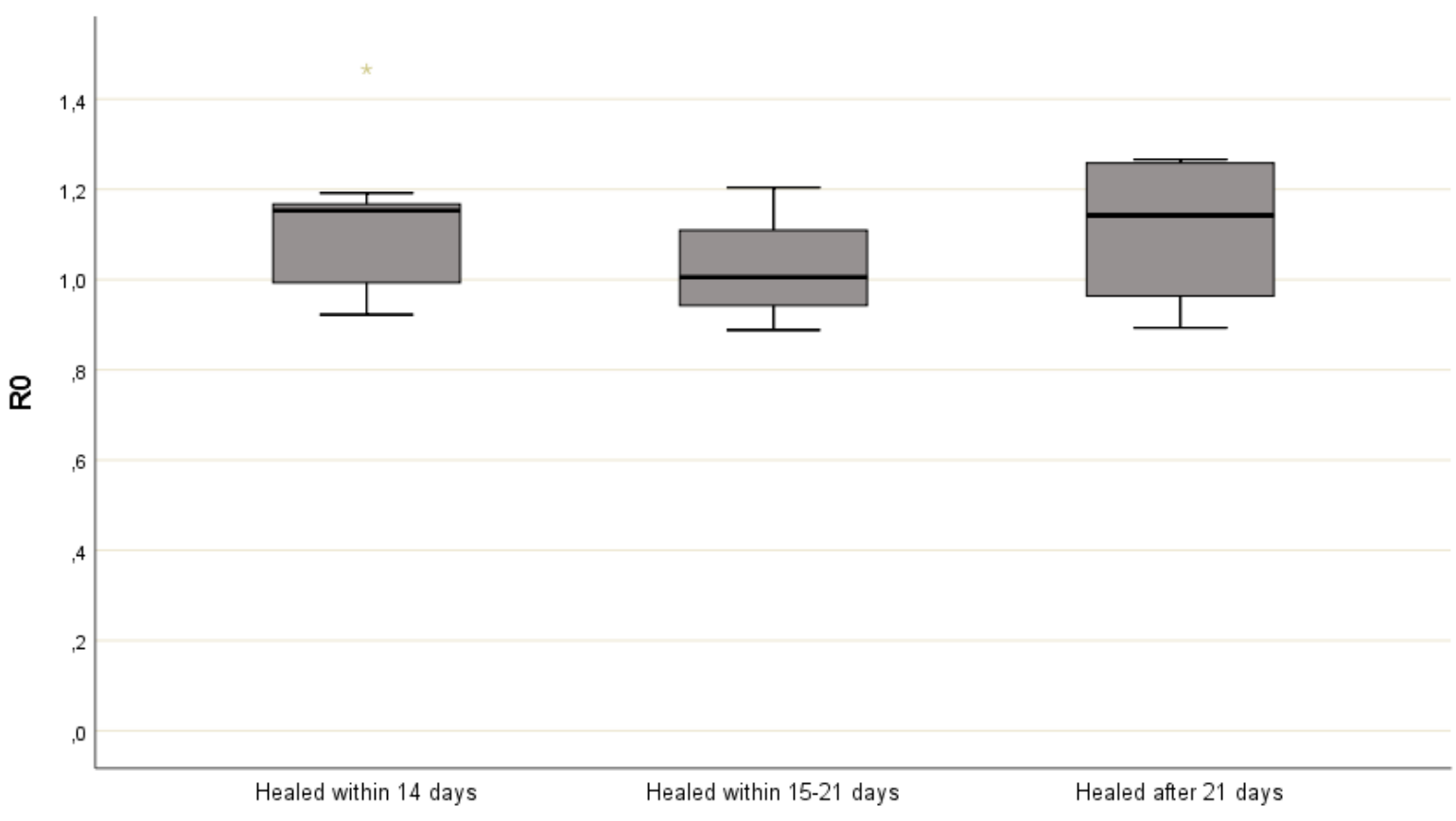

Figure 3. R0 values for the different "healing groups". The lower the value, the higher the firmness of the skin. Median, IQR and range are displayed.

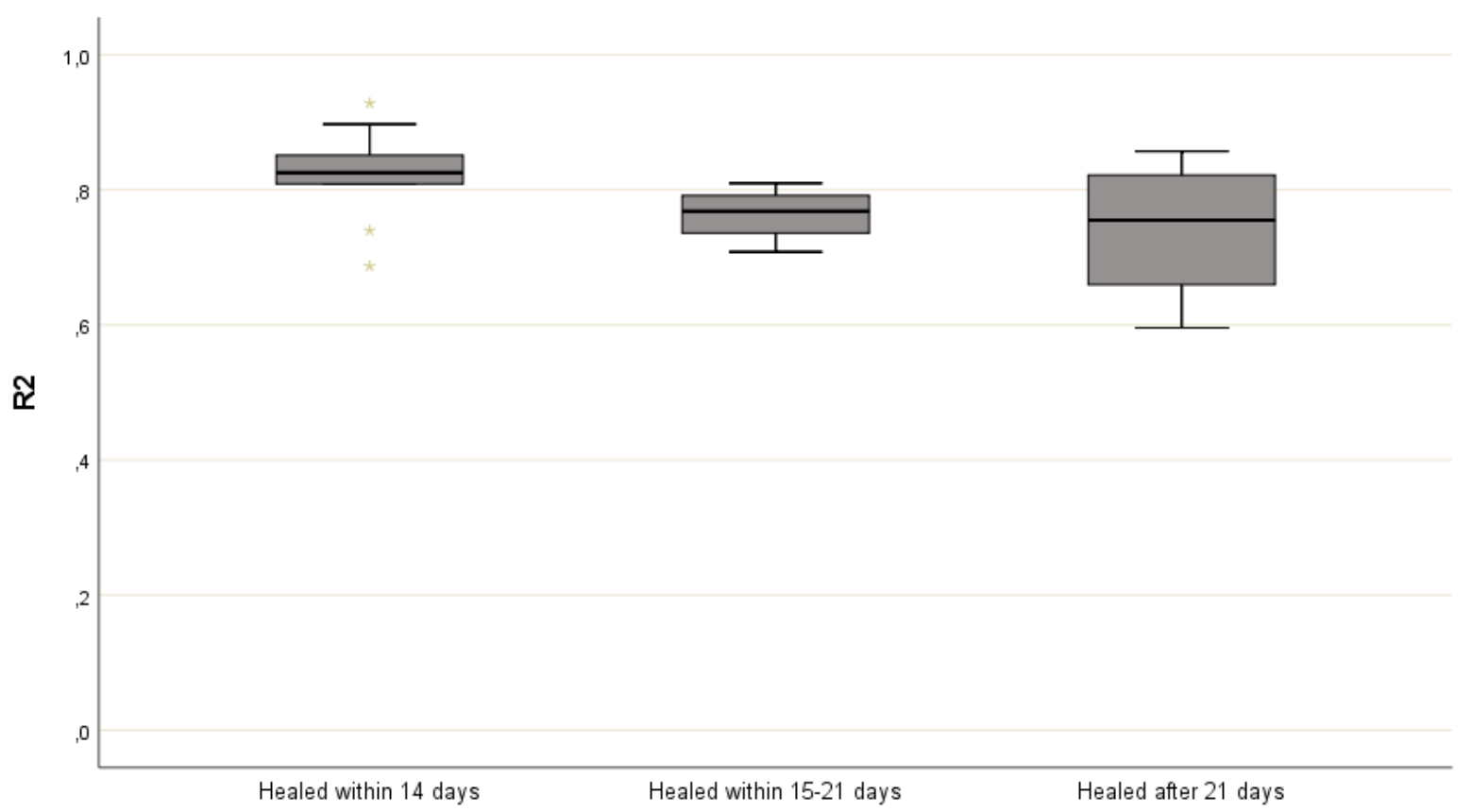

Figure 4. R2 values for the different "healing groups". The closer the value is to $1(100 \%)$, the more elastic the skin. Median, IQR and range are displayed. 


\section{Revised supplemental figures and tables}

\begin{tabular}{|c|c|c|c|c|}
\hline \multicolumn{5}{|c|}{ Supplemental table 1. POSAS scores given by Observer by treatment group } \\
\hline & Hydrofiber $(\mathrm{n}=5)$ & Polyurethane foam $(\mathrm{n}=6)$ & Porcine xenograft $(\mathrm{n}=6)$ & $\mathrm{p}$ value \\
\hline \multicolumn{5}{|c|}{ Vascularity } \\
\hline Score 1. & 4 & 5 & 6 & \\
\hline Score 2. & 1 & 1 & 0 & 0.735 \\
\hline \multicolumn{5}{|c|}{ Pigmentation } \\
\hline Score 1 & 0 & 0 & 1 & \\
\hline Score 2 & 1 & 1 & 1 & \\
\hline Score 3 & 3 & 3 & 2 & \\
\hline Score 4 & 1 & 2 & 0 & \\
\hline Score 5 & 0 & 0 & 2 & 0.758 \\
\hline \multicolumn{5}{|l|}{ Thickness } \\
\hline Score 1 & 4 & 5 & 5 & \\
\hline Score 2 & 0 & 1 & 1 & \\
\hline Score 4 & 1 & 0 & 0 & 1.0 \\
\hline \multicolumn{5}{|l|}{ Relief } \\
\hline Score 1 & 4 & 5 & 6 & \\
\hline Score 2 & 0 & 1 & 0 & \\
\hline Score 3 & 1 & 0 & 0 & 0.735 \\
\hline \multicolumn{5}{|l|}{ Pliability } \\
\hline Score 1 & 5 & 6 & 6 & . \\
\hline \multicolumn{5}{|c|}{ Surface area } \\
\hline Score 2 & 0 & 0 & 1 & \\
\hline Score 4 & 0 & 0 & 1 & \\
\hline Score 5 & 0 & 1 & 0 & \\
\hline Score 6 & 1 & 0 & 1 & \\
\hline Score 7 & 0 & 1 & 0 & \\
\hline Score 8 & 1 & 1 & 0 & \\
\hline Score 9 & 3 & 3 & 3 & 1.0 \\
\hline
\end{tabular}


Supplemental Table 2. All POSAS scores, Observer scale items and total score in Patients scale

\begin{tabular}{|c|c|c|c|c|c|c|c|c|c|}
\hline \multirow{2}{*}{\multicolumn{2}{|c|}{ Hydrofiber }} & \multirow[t]{2}{*}{ Vascularity } & \multirow[t]{2}{*}{ Pigmentation } & \multirow[t]{2}{*}{$\underline{\text { Thickness }}$} & \multirow[t]{2}{*}{$\underline{\text { Relief }}$} & \multirow[t]{2}{*}{$\underline{\text { Pliability }}$} & \multirow[t]{2}{*}{$\underline{\text { Surface area }}$} & \multirow[t]{2}{*}{$\underline{\text { Total score }}$} & \multirow[t]{2}{*}{ Patient's Total score * } \\
\hline & & & & & & & & & \\
\hline Patient & 1 & 1 & 2 & 1 & 1 & 1 & 6 & 12 & 14 \\
\hline & 2 & 1 & 3 & 1 & 1 & 1 & 8 & 15 & 14 \\
\hline & 3 & 1 & 3 & 1 & 1 & 1 & 9 & 16 & 9 \\
\hline & 4 & 1 & 4 & 1 & 1 & 1 & 9 & 17 & 15 \\
\hline & 5 & 2 & 3 & 4 & 3 & 1 & 9 & 22 & 12 \\
\hline \multicolumn{10}{|c|}{ Polyurethane foam } \\
\hline Patient & 1 & 1 & 2 & 1 & 1 & 1 & 5 & 11 & 15 \\
\hline & 2 & 1 & 3 & 1 & 1 & 1 & 8 & 15 & 19 \\
\hline & 3 & 1 & 3 & 1 & 1 & 1 & 9 & 16 & 15 \\
\hline & 4 & 1 & 3 & 1 & 1 & 1 & 9 & 16 & 10 \\
\hline & 5 & 1 & 4 & 1 & 1 & 1 & 9 & 17 & 9 \\
\hline & 6 & 2 & 4 & 2 & 2 & 1 & 7 & 18 & 16 \\
\hline \multicolumn{10}{|c|}{$\underline{\text { Porcine xenograft }}$} \\
\hline Patient & 1 & 1 & 1 & 2 & 1 & 1 & 2 & 8 & 7 \\
\hline & 2 & 1 & 3 & 1 & 1 & 1 & 6 & 13 & 19 \\
\hline & 3 & 1 & 3 & 1 & 1 & 1 & 9 & 16 & 10 \\
\hline & 4 & 1 & 2 & 1 & 1 & 1 & 4 & 17 & 11 \\
\hline & 5 & 1 & 5 & 1 & 1 & 1 & 9 & 18 & 21 \\
\hline & 6 & 1 & 5 & 1 & 1 & 1 & 9 & 18 & 25 \\
\hline
\end{tabular}

Numbers are scores given for each item on the POSAS (scale 1-10) * Results from previous study (reference 2). 
Supplemental table 3. Mean difference values for Cutometer measurements between donor site and healthy skin within groups

\begin{tabular}{lcccc}
\hline & Hydrofibre & Polyurethane foam & Porcine xenograft & p values \\
R0 & $0.14(0.14)$ & $-0.02(0.20)$ & $-0.04(0.27)$ & 0.356 \\
R2 & $0.02(0.07)$ & $-0.02(0.11)$ & $-0.03(0.10)$ & 0.740 \\
F1 & $0.01(0.03)$ & $-0.02(0.03)$ & $-0.03(0.03)$ & 0.115 \\
\hline
\end{tabular}

Data are mean values and standard deviations. . Lower R0 indicates higher firmness, higher R2 indicates higher elasticity, and lower F1 indicates higher elasticity.

Supplemental table 4. Cutometer measurements on donor sites grouped by healing times

\begin{tabular}{l|cccc}
\hline & $\leq \mathbf{1 4}$ days & $\mathbf{1 5 - 2 1}$ days & $>\mathbf{2 1}$ days & p values \\
R0 & $1.06(0.14)$ & $1.07(0.13)$ & $1.19(0.21)$ & 0.374 \\
R2 & $0.81(0.06)$ & $0.75(0.06)$ & $0.80(0.14)$ & 0.524 \\
F1 & $0.13(0.03)$ & $0.12(0.01)$ & $0.13(0.05)$ & 0.668 \\
\hline
\end{tabular}

Data are mean values and standard deviations. Lower R0 indicates higher firmness, higher R2 indicates higher elasticity, and lower F1 indicates higher elasticity. 


\section{POSAS Observer scale}

The Patient and Observer Scar Assessment Scale v2.0/ EN

\begin{tabular}{l} 
Date of examination: \\
\hline Observer: \\
\hline Location: \\
Research/study:
\end{tabular}

Name of patient:

Date of birth:

Identification number:

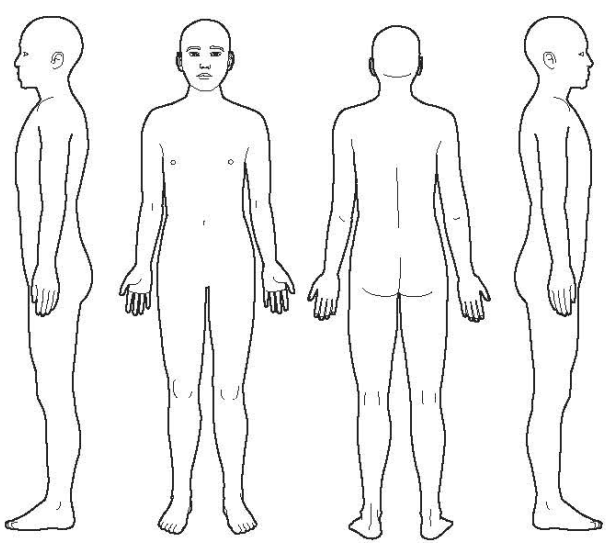

$\sum_{\infty} 9$

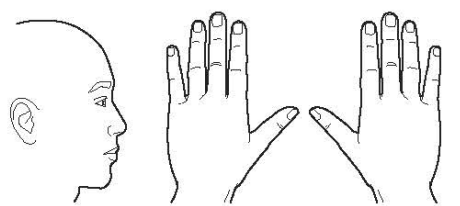
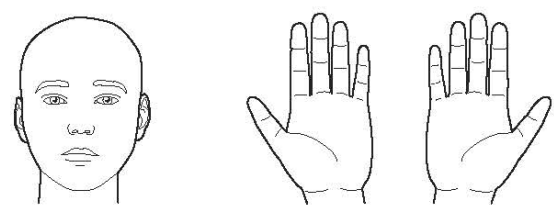

$1=$ normal skin

worst scar imaginable $=10$

PARAMETER

OVERALL OPINION

Explanation

The observer scale of the POSAS consists of six items (va scularity, pigmentation, thickness, relief, pliability and surface area).

All items are scored on a scale ranging from 7 ("like normal skin")

to 10 ('worst scar imaginable').

The sum of the six items results in a total score of the POSAS observer

scale. Categories boxes a readded for each item. Fu rthermore, an overal

opinion is scored on a scale ranging from 1 to 10

All parameters should preferably be compared to normal skin on a comparable anatomic location.

\author{
Explanatory notes on the items: \\ - vas cularitr Presence of vessels in scar tissue assessed by the amount \\ of redness, tested by the amount of blood return after blanching with \\ piece of Plexiglas \\ - PIGMentarion Brownish coloration of the scar by pigment (melanin) \\ applyplexiglas to the skin with moderate pressure to eliminatethe \\ effect of vascularity \\ - THICKNEss Average distance between the subcutical-dermal border \\ and the epidermal surface of the scar \\ - RELEF The extent to which surface irregularities are present \\ (preferably compared with adjacent normalskin) \\ - PLIABILITY suppleness of the scar tested by wrinkling the scar between \\ the thumb and index finger \\ - sURF ACE AREA Surface area of the scar in relation to the original wound area
}

Supplemental Figure 1. Reprinted with permission from the POSAS group, August 26, 2019. Copyright holders Copyright holders P.P.M. van Zuijlen, Beverwijk-NL. 


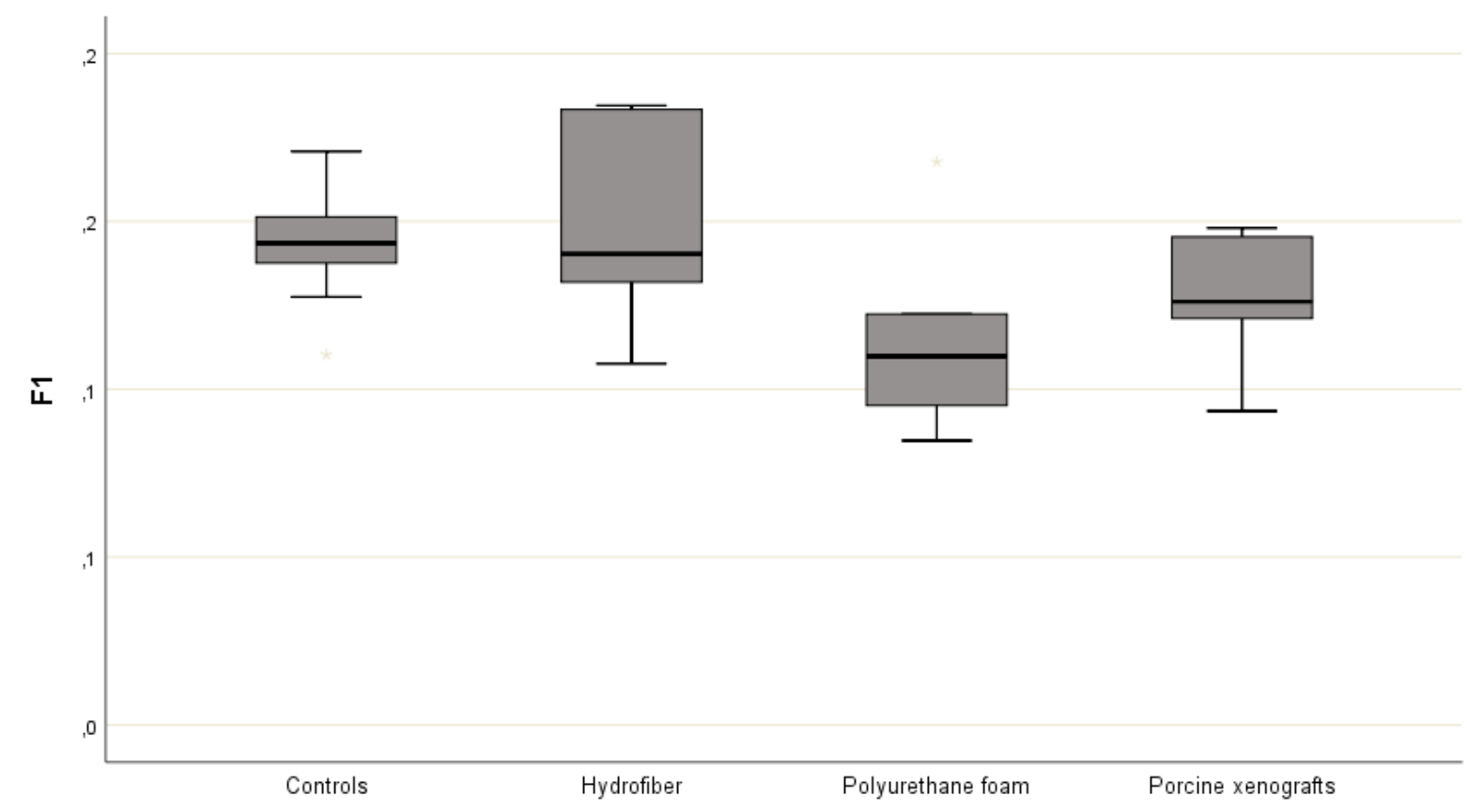

Supplemental figure 2. F1 values for all controls and the three dressing groups. The closer to 0 the value is, the more elastic the skin. Median, IQR and range are displayed.

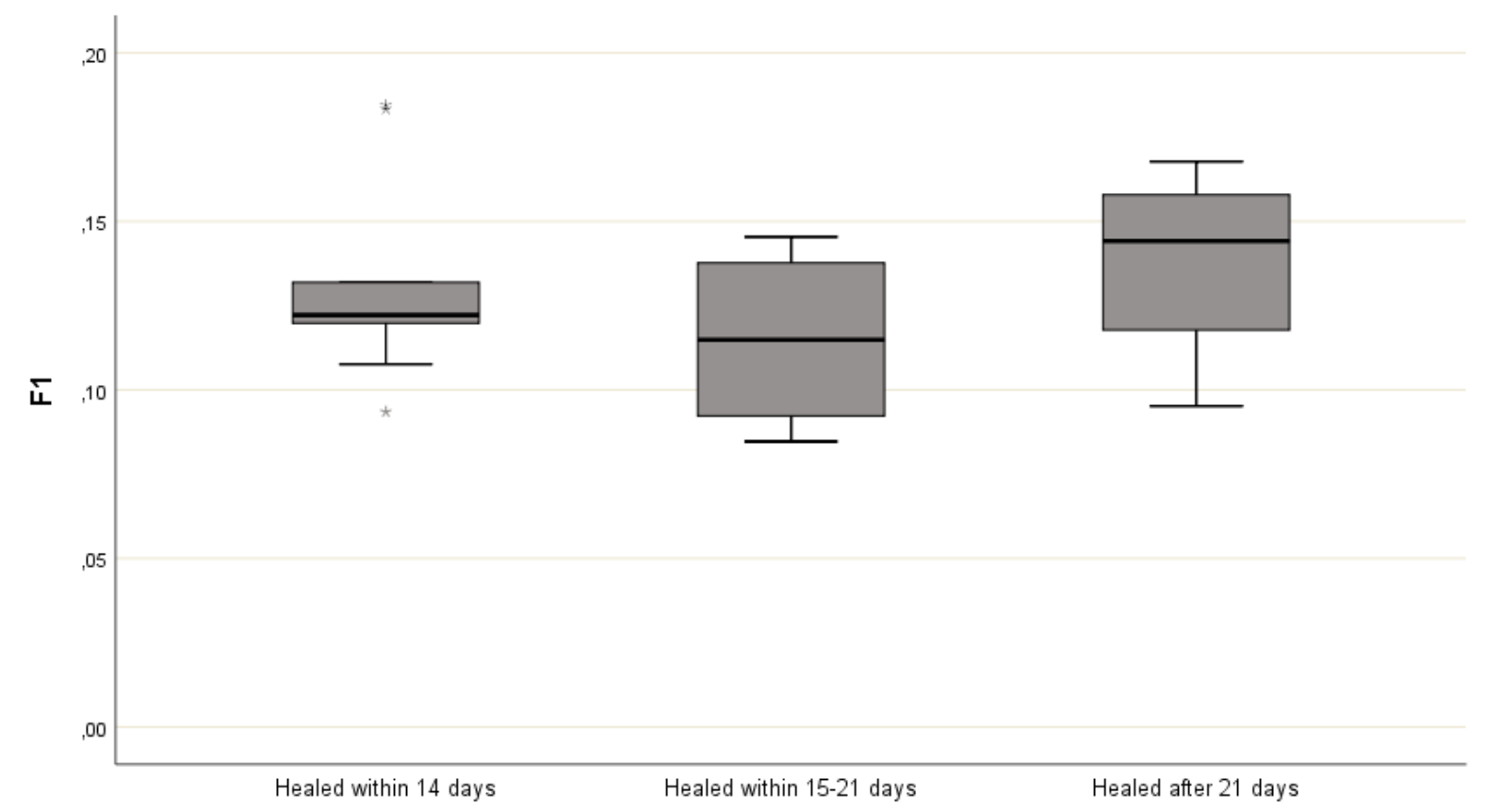

Supplemental figure 3. F1 values for the different "healing groups". The closer to 0 the value is, the more elastic the skin. Median, IQR and range are displayed. 